\title{
THE EQUIVALENCE OF VARIOUS LIPSCHITZ CONDITIONS ON THE WEIGHTED MEAN OSCILLATION OF A FUNCTION
}

\author{
EDWARD P. LOTKOWSKI AND RICHARD L. WHEEDEN ${ }^{1}$
}

ABSTRACT. The main result establishes the equivalence of various Lipschitzlike conditions on the weighted mean oscillation over cubes of a function. Previously known results are obtained as special cases.

Introduction. In [3], John and Nirenberg proved that if $f$ is a function of bounded mean oscillation, i.e., if

$$
\int_{I}\left|f(x)-f_{I}\right| d x \leqslant c|I|, \quad f_{I}=\frac{1}{|I|} \int_{I} f(t) d t,
$$

for all cubes $I \subset R^{n}$, with $c$ independent of $I$, then there exist positive constants $c_{1}$ and $c_{2}$ depending only on $n$ such that

$$
\left|\left\{x \in I:\left|f(x)-f_{I}\right|>\alpha\right\}\right| \leqslant c_{1} e^{-c_{2} \alpha / c}|I|
$$

for all $I$ and $\alpha>0$. In [7] Muckenhoupt and Wheeden derived an analogous result for functions of weighted bounded mean oscillation, by which we mean those $f$ with

$$
\int_{I}\left|f(x)-f_{I}\right| d x \leqslant c \int_{I} w(x) d x
$$

for all $I$, where $w$ is nonnegative and satisfies appropriate conditions.

Meyers [5] and Campanato [1] showed independently that the condition

$$
\int_{I}\left|f(x)-f_{I}\right| d x \leqslant c|I|^{1+\varepsilon}
$$

for an $\varepsilon$ satisfying $0<\varepsilon \leqslant 1$ is equivalent (after redefining $f$ in a set of measure 0 ) to Lipschitz continuity of order $\varepsilon$. Two different weighted versions of this result were then obtained by Cuerva [2] and Lotkowski [4]. Our main theorem generalizes and unifies these latter results, as we shall indicate below.

We first list some definitions. If $\mu$ is a Borel measure which is positive and

Received by the editors April 2, 1976.

AMS (MOS) subject classifications (1970). Primary 26A16.

${ }^{1}$ Research supported in part by NSF-MPS 75-07596. 
finite on cubes and $f$ is locally integrable with respect to $\mu$, then $f_{I, d \mu}$ (or simply $f_{I}$ ) will denote the average of $f$ over $I$ with respect to $\mu$ :

$$
f_{I}=f_{I, d \mu}=\frac{1}{\mu(I)} \int_{I} f(x) d \mu(x) .
$$

For $a>0, a I$ will denote the cube concentric with $I$ whose edge length is $a$ times that of $I$. We shall be concerned only with measures which satisfy the doubling condition

$$
\mu(2 I) \leqslant c \mu(I)
$$

for every $I$, with $c$ independent of $I$. It is an easy consequence of (1) that two cubes of equal size whose centers are at a distance bounded by a fixed multiple of their edge length have equivalent $\mu$ measure. For $1<p<\infty$, a nonnegative function $u$ is said to belong to $A_{p}(d \mu)$ if there is a constant $c$ such that for all $I$

$$
\left(\frac{1}{\mu(I)} \int_{I} u(x) d \mu(x)\right)\left(\frac{1}{\mu(I)} \int_{I} u(x)^{-1 /(p-1)} d \mu(x)\right)^{p-1} \leqslant c .
$$

Similarly, $u$ is said to belong to $A_{1}(d \mu)$ if

$$
u_{\mu}^{*}(x) \leqslant c u(x) \text { a.e. }(d \mu)
$$

with $c$ independent of $x$, where $u_{\mu}^{*}$ is the Hardy-Littlewood maximal function of $u$ with respect to $d \mu$ :

$$
u_{\mu}^{*}(x)=\sup \left\{\frac{1}{\mu(I)} \int_{I} u(t) d \mu(t): x \in I\right\} .
$$

$F(I)$ will denote a positive function of cubes for which there exist constants $\alpha, \beta$ and $c$ such that $1<\alpha<\beta, c>0$ and

$$
\begin{gathered}
F(I) \leqslant c F(J) \quad \text { if } I \subset J, \\
\alpha F(I) \leqslant F(2 I) \leqslant \beta F(I) .
\end{gathered}
$$

It is crucial for what follows that $\alpha$ strictly exceed 1 . An example of such an $F$ is

$$
F(I)=\left[\mu_{1}(I)\right]^{\varepsilon_{1}} \cdots\left[\mu_{m}(I)\right]^{\varepsilon_{m}}
$$

with $\mu_{j}$ satisfying (1) and $\varepsilon_{j}>0$ for $1 \leqslant j \leqslant m$.

We shall often use the same letter $c$ to denote different constants which are independent of $x$ and $I$. For a function $g$ which is nonzero a.e., $g^{-1}$ will denote the function $1 / g$.

Our main result is given by the following theorem.

THEOREM. Suppose that $g$ is nonnegative and locally integrable with respect to 
$\mu$, that the measure gd $\mu$ satisfies (1), and that $F$ satisfies (4) and (5). If $1 \leqslant p<\infty$ and $g^{-1} \in A_{p}(g d \mu)$, then the condition

$$
\int_{I}\left|f(x)-f_{I}\right| g(x) d \mu(x) \leqslant c \mu(I) F(I), \quad f_{I}=f_{I, g d \mu},
$$

is equivalent to

$$
\int_{I}\left\{\left|f(x)-f_{I}\right| g(x)\right\}^{p^{\prime}} d \mu(x) \leqslant c \mu(I) F(I)^{p^{\prime}}, \quad 1 / p+1 / p^{\prime}=1 .
$$

In case $p=1$, we have $p^{\prime}=\infty$, and (7) should be interpreted in the $L^{\infty}$ sense:

$$
\text { ess } \sup _{I}\left\{\left|f(x)-f_{I}\right| g(x)\right\} \leqslant c F(I),
$$

the ess sup being taken with respect to $d \mu$, or equivalently $g d \mu$.

Before proceeding with the proof, we list two important special cases. First, given $\varepsilon>0$ and a weight function $w$, we choose

$$
\mu(I)=\int_{I} w(x) d x, \quad F(I)=\left[\int_{I} w(x) d x\right]^{\varepsilon}, \quad g(x)=w(x)^{-1},
$$

obtaining that for $1<p<\infty$, the condition

$$
\int_{I}\left|f(x)-f_{I}\right| d x \leqslant c\left[\int_{I} w(x) d x\right]^{1+\varepsilon}, \quad f_{I}=f_{I, d x},
$$

is equivalent to

$$
\int_{I}\left|f(x)-f_{I}\right|^{p^{\prime}} w(x)^{-1 /(p-1)} d x \leqslant c\left[\int_{I} w(x) d x\right]^{1+\varepsilon p^{\prime}}, \quad 1 / p+1 / p^{\prime}=1,
$$

provided that $w \in A_{p}(d x)$. If $w \in A_{1}(d x)$, (9) is equivalent to

$$
\text { ess } \sup _{I}\left\{\left|f(x)-f_{I}\right| w(x)^{-1}\right\} \leqslant c\left[\int_{I} w(x)\right]^{\varepsilon},
$$

the ess sup being with respect to Lebesgue measure. Results of this type were proved in [2], using duality methods.

Taking $\mu$ and $\nu$ to satisfy (1), $F(I)=\nu(I)^{\varepsilon}$ and $g(x) \equiv 1$, one obtains that for any $p \geqslant 1$, the condition

$$
\int_{I}\left|f(x)-f_{I}\right|^{p^{\prime}} d \mu(x) \leqslant c \mu(I) \nu(I)^{\varepsilon p^{\prime}}, \quad f_{I}=f_{I, d \mu}
$$

is equivalent to

$$
\left|f(x)-f_{I}\right| \leqslant c \nu(I)^{\varepsilon} \quad \text { for a.e. }(d \mu) x \in I,
$$

since in this case $g$ trivially satisfies the $A_{p}$ condition for any $p \geqslant 1$. This may be found in [4]. 
Proof of the Theorem. The hard part of the proof is the implication (6) $\Rightarrow$ (7), the opposite one being an immediate corollary of Hölder's inequality. The proof we give is based on the methods used in [1] and [4]. We would like to thank Professor C. Fefferman for a useful comment concerning the proof of Lemma 1.

Lemma 1. Let $\mu$ and $\nu$ satisfy (1), and F satisfy (4) and (5). If

$$
\int_{I}\left|f(x)-f_{I}\right| d \nu(x) \leqslant c \mu(I) F(I), \quad f_{I}=f_{I, d v},
$$

then for a.e. $(d v) x \in I$,

$$
\left|f(x)-f_{I}\right| \leqslant c F(I) \sup \{(\mu(J) / \nu(J)): x \in J \subset 2 I\} .
$$

Proof. For $x \in I$, let $I_{x}$ denote the cube centered at $x$ with the same edge length as $I$. We first claim that

$$
\left|f_{I}-f_{I_{x}}\right| \leqslant c F(I) \frac{\mu(I)}{\nu(I)} .
$$

In fact,

$$
\begin{aligned}
\left|f_{I}-f_{I_{x}}\right| & \leqslant\left|f_{I}-f_{2 I}\right|+\left|f_{2 I}-f_{I_{x}}\right| \\
& =\left|\frac{1}{\nu(I)} \int_{I}\left\{f(t)-f_{2 I}\right\} d v(t)\right|+\left|\frac{1}{\nu\left(I_{x}\right)} \int_{I_{x}}\left\{f(t)-f_{2 I}\right\} d \nu(t)\right| .
\end{aligned}
$$

Since both $I$ and $I_{x}$ lie in $2 I$, and $\nu$ satisfies (1), each of the last two terms is majorized by

$$
\frac{c}{\nu(I)} \int_{2 I}\left|f(t)-f_{2 I}\right| d \nu(t) .
$$

Using (10) to estimate the last integral, and then applying (1), (4) and (5), we obtain (12).

Next, we will show that for a.e. $(d v) x \in I$,

$$
\left|f(x)-f_{I_{x}}\right| \leqslant c F(I) \sup \{(\mu(J) / \nu(J)): x \in J \subset 2 I\} .
$$

To see this, set $I_{k}=2^{-k} I_{x}$, and note that for a.e. $(d v) x \in I$, differentation of the integral gives

$$
\left|f(x)-f_{I_{x}}\right| \leqslant \sum_{k=0}^{\infty}\left|f_{I_{k}}-f_{I_{k+1}}\right| .
$$

However,

$$
\begin{aligned}
\left|f_{I_{k}}-f_{I_{k+1}}\right| & \leqslant \frac{1}{\nu\left(I_{k+1}\right)} \int_{I_{k+1}}\left|f(t)-f_{I_{k}}\right| d \nu(t) \\
& \leqslant \frac{c}{\nu\left(I_{k}\right)} \int_{I_{k}}\left|f(t)-f_{I_{k}}\right| d \nu(t)
\end{aligned}
$$


by (1) and the fact that $I_{k+1} \subset I_{k}$. Hence, by (10),

$$
\left|f_{I_{k}}-f_{I_{k+1}}\right| \leqslant \frac{c}{\nu\left(I_{k}\right)} \mu\left(I_{k}\right) F\left(I_{k}\right) .
$$

Since $2^{k} I_{k}=I_{x}$, (4) and (5) imply that $F\left(I_{k}\right) \leqslant c \alpha^{-k} F(I)$. Combining estimates, we obtain that for a.e. $(d v) x \in I$,

$$
\begin{aligned}
\left|f(x)-f_{I_{x}}\right| & \leqslant c F(I) \sum_{k=0}^{\infty} \alpha^{-k} \frac{\mu\left(I_{k}\right)}{\nu\left(I_{k}\right)} \\
& \leqslant c F(I)\left\{\sup \frac{\mu(J)}{\nu(J)}\right\} \sum_{k=0}^{\infty} \alpha^{-k},
\end{aligned}
$$

where the sup is taken over all cubes $J$ such that $x \in J$ and $J \subset 2 I$. Since $\alpha>1$, the series converges and (13) follows. Combining (12) and (13) concludes the proof of the lemma.

We shall also use the following fact.

Lemma 2. Let $\nu$ be a Borel measure which satisfies (1), and let $f_{\nu}^{*}$ denote the Hardy-Littlewood maximal function of $f$ with respect to $\nu$ :

$$
f_{\nu}^{*}(x)=\sup \left\{\frac{1}{\nu(I)} \int_{I}|f(t)| d \nu(t): x \in I\right\} .
$$

If $u \in A_{p}(d v), 1<p<\infty$, then there is a constant $c$, independent of $f$, such that

$$
\int_{R^{n}} f_{\nu}^{*}(x)^{p} u(x) d v(x) \leqslant c \int_{R^{n}}|f(x)|^{p} u(x) d v(x) .
$$

This is a restatement of Theorem 7 of [6].

We now turn to the proof of the theorem, first showing that the assumptions on $g d \mu$ and $g^{-1}$ imply that $\mu$ satisfies (1). Hölder's inequality and $g^{-1}$ $\in A_{p}(g d \mu)$ imply

$$
\left[\int_{I} g d \mu\right]^{p} \leqslant \mu(I)\left[\int_{I} g^{p /(p-1)} d \mu\right]^{p-1} \leqslant c\left[\int_{I} g d \mu\right]^{p} .
$$

Since the same relation holds for $2 I$,

$$
\mu(2 I)\left[\int_{I} g^{p /(p-1)} d \mu\right]^{p-1} \leqslant c\left[\int_{2 I} g d \mu\right]^{p} \leqslant c\left[\int_{I} g d \mu\right]^{p},
$$

by (1) for $g d \mu$. Hence, by the first inequality (14), $\mu(2 I) \leqslant c \mu(I)$.

If we set $d \nu=g d \mu$, then hypothesis (6) of the theorem is the same as (10) of Lemma 1. Hence, by Lemma 1, for a.e. $(d v) x \in I$,

$$
\left|f(x)-f_{I}\right| \leqslant c F(I) \sup \{(\mu(J) / \nu(J)): x \in J \subset 2 I\},
$$


where $f_{I}=f_{I, d \nu}$. If $\chi_{2 I}$ denotes the characteristic function of $2 I$, it follows from the formula $\mu(J)=\int_{J} g^{-1} d \nu$ that

$$
\left|f(x)-f_{I}\right| \leqslant c F(I)\left[g^{-1} \chi_{2 I}\right]_{\nu}^{*}(x) \text { for a.e. }(d v) x \in I .
$$

For $1<p<\infty$, multiply both sides of (15) by $g(x)$, raise the result to the power $p^{\prime}$, and then integrate over $I$ with respect to $d \mu$, obtaining

$$
\int_{I}\left\{\left|f-f_{I}\right| g\right\}^{p^{\prime}} d \mu \leqslant c F(I)^{p^{\prime}} \int_{R^{n}}\left\{\left[g^{-1} \chi_{2 I}\right]_{\nu}^{*}\right\}^{p^{\prime}} g^{p^{\prime}-1} d \nu .
$$

It is easy to see that the assumption $g^{-1} \in A_{p}(d \nu)$ is equivalent to $g^{p^{\prime}-1}$ $\in A_{p^{\prime}}(d \nu)$. Hence, Lemma 2 shows that the expression on the right in (16) is at most

$$
c F(I)^{p^{\prime}} \int_{2 I} g^{-p^{\prime}} g^{p^{\prime}-1} d \nu=c F(I)^{p^{\prime}} \mu(2 I) .
$$

Since $\mu$ satisfies (1), we obtain

$$
\int_{I}\left\{\left|f-f_{I}\right| g\right\}^{p^{\prime}} d \mu \leqslant c F(I)^{p^{\prime}} \mu(I),
$$

as desired. If $p=1$, the hypothesis $g^{-1} \in A_{1}(d \nu)$ gives

$$
\left[g^{-1}\right]_{\nu}^{*} \leqslant c g^{-1} \text { a.e. }
$$

Combining this with (15) proves (8). Thus, the proof of the theorem is complete.

As a final remark, we note that the proof given above does not yield the results on bounded mean oscillation mentioned previously, since these correspond to $F(I) \equiv 1$, which fails to satisfy (5) ( $\alpha$ must strictly exceed 1 there, in order that the series in Lemma 1 converge).

\section{REFERENCES}

1. S. Campanato, Proprietà di hölderianità di alcune classi di funzioni, Ann. Scuola Norm Sup. Pisa (3) 17 (1963), 175-188. MR 27 \#6119.

2. J.-G. Cuerva, Weighted $H^{p}$ spaces, Ph.D. dissertation, Washington Univ., St. Louis, Mo., 1975.

3. F. John and L. Nirenberg, On functions of bounded mean oscillation, Comm. Pure Appl. Math. 14 (1961), 415-426. MR 24 \# A1348.

4. E. P. Lotkowski, Lipschitz spaces with weights, Ph.D. dissertation, Rutgers Univ., New Brunswick, N.J., 1975.

5. N. G. Meyers, Mean oscillation over cubes and Hölder continuity, Proc. Amer. Math. Soc. 15 (1964), 717-721. MR 29 \#5969.

6. B. Muckenhoupt, Weighted norm inequalities for the Hardy maximal function, Trans. Amer. Math. Soc. 165 (1972), 207-226. MR 45 \#2461.

7. B. Muckenhoupt and R. L. Wheeden, Weighted bounded mean oscillation and the Hilbert transform, Studia Math. 54 (1976), 221-237.

Department of Mathematics, Purdue University, West Lafayette, Indiana 47907

Department of Mathematics, Rutgers University, New Brunswick, New Jersey 08903 (Current address of R. L. Wheeden)

Current address (E. P. Lotkowski): Prudential Property and Casualty Insurance Company, One Woodbridge Center, Woodbridge, New Jersey 07095 